\title{
Abducens palsy after temporomandibular joint arthroscopy: a rare complication
}

\author{
Fábio Ricardo Loureiro Sato ${ }^{1} \cdot$ Rodrigo Corrêa Tavares ${ }^{2}$
}

Received: 9 February 2016 / Accepted: 20 April 2016/Published online: 4 May 2016

(C) Springer-Verlag Berlin Heidelberg 2016

\begin{abstract}
Background Arthroscopy of the temporomandibular joint (TMJ) is a minimally invasive and safe procedure; nevertheless, some accidents and complications have been described in the literature.

Case report The purpose of this article is to describe a case report of a patient that progressed to paralysis of the abducens nerve after a TMJ arthroscopy procedure.
\end{abstract}

Keywords Tempomandibular joint · Arthroscopy Arthroplasty $\cdot$ Abducens nerve $\cdot$ Ophthalmoplegia $\cdot$ Diplopia

\section{Introduction}

Arthroscopy has been an increasingly used procedure to treat temporomandibular disorders. It is considered a not only diagnostic but also therapeutic minimally invasive technique that can be performed from lysis and lavage procedures to arthroscopic disc repositioning, in hands of an experienced surgeon, helping reduce pain complaints in a significant number of cases, especially those refractory to conservative treatment. When arthroscopy is compared with other surgical procedures, it is considered a safe procedure with few accidents and complications, like pain, swelling, vascular problems,

Fábio Ricardo Loureiro Sato

fabio.sato@ict.unesp.br

1 Oral and Maxillofacial Surgery Area, College of Dentistry, State University of São Paulo-UNESP, Av. Eng. Francisco José Longo, 777, São José dos Campos 12245-000, Brazil

2 Private Practice, São Paulo, SP, Brazil perforation of the middle cranial fossa, hemorrhage, nerve damage, and instrument breakage [1]. Only one case reporting the oculomotor and trochlear nerve palsy after a TMJ arthroscopy was found in the literature review [2]. This latter complication involving the innervation of ocular muscles presents more case reports related to Le Fort I osteotomy in literature [3-5], although being rare, as well as post-traumatic lesions [6]. No article reporting the paralysis of the abducens nerve as a complication of arthroscopy was found in the literature, as observed in this case report. Informed consent was obtained from the patient in this case report.

\section{Case report}

Female patient, 33, melanodermic, complaining of bilateral pain in the TMJ (VAS $=6$ ), presented episodes of joint clicking on the left side with mouth opening with an "S" deviation. According to the patient, this situation had a 1year history, and, after clinical assessment, she was referred to conservative treatment with the use of occlusal splints and physical therapy. However, after 6 months of conservative treatment, she had gotten worse in terms of painful condition, with bilaterally increased pain (VAS $=8$ ). A magnetic resonance imaging (MRI) of the bilateral temporomandibular joint (TMJ) was requested, which found an anterior disc displacement with reduction on the right, and disc at the normal position with effusion on the left side. Based on this clinical picture, arthroscopic lysis and lavage of the left and arthroscopic discopexy were indicated for the right side. The patient has no relevant medical history, or history of ophthalmologic interventions, including no changes regarding eye movement before the surgical procedure.

The procedure was performed under general anesthesia without complications, with a double puncture technique- 
inflow by the posterolateral portal and outflow by the anterolateral portal (first infiltration with $3 \mathrm{ml}$ of lidocaine $2 \%$ without adrenaline in the superior joint space of the TMJ and after continuous perfusion of about $500 \mathrm{ml}$ lactated Ringer's solution). Before injecting the anesthetics, aspiration was done with negative result, demonstrating that the needle was in the superior joint space. A 1.9-mm Storz Arthroscope and Cannulas (Karl Storz GmbH, Tuttlingen, Germany) were used. For the left side, only lysis and lavage were performed. For the right side, radiofrequency (Arthrocare Corp., TX, USA) capsulotomy, and myotomy for anterior release of the lateral pterygoid muscle and posterior repositioning of the articular disc with suture were performed under the McCain's technique [7] through a third gate, using polydioxanone suture/PDS 2.0 (Ethicon Inc., NJ, USA). No intra-articular drugs were injected after the procedure. The total duration of the procedure was $45 \mathrm{~min}$, without any transoperative incident, and almost 450 of $500 \mathrm{ml}$ of the lactated Ringer's solution injected was recovered through outflow ports. No significant swelling could be identified in the face or in the oropharynx.

After the surgical procedure is finished, the patient presented diplopia in all except levoversion field of vision, along with the presence of convergent strabismus in the primary position of the right eye (Fig. 1), limitation of movement in dextroversion on the same side (Fig. 2), and isochoric and photo reactive pupils in the immediate postoperative period. The patient was subject the same day to neurological evaluation. The neurological evaluation ordered MRI and CT scans of TMJ and cranium in the same day of the surgical procedure, with emphasis on temporal bones and excluded the possibility of a trauma or tumor in the cavernous sinus, increased intracranial pressure due to trauma or a stroke. Neurosurgery team requested immediately using cytidine + uridine + hydroxocobalamin $\left(E T N A^{\circledR}\right.$, Gross, Rio de Janeiro, Brazil) for 50 days. Four days after the surgery, a new neurological evaluation was performed, and the start of laser therapy

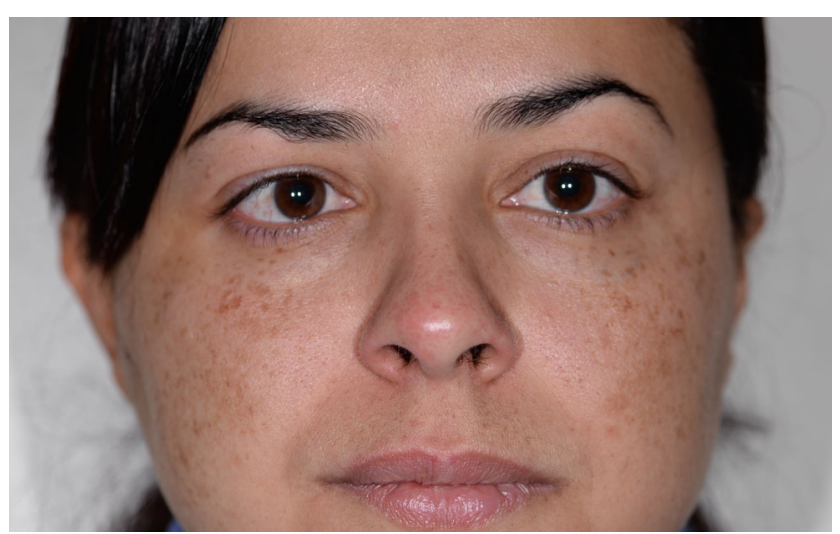

Fig. 1 Extraoral photo of the patient showing convergent strabismus in the primary position of the right eye

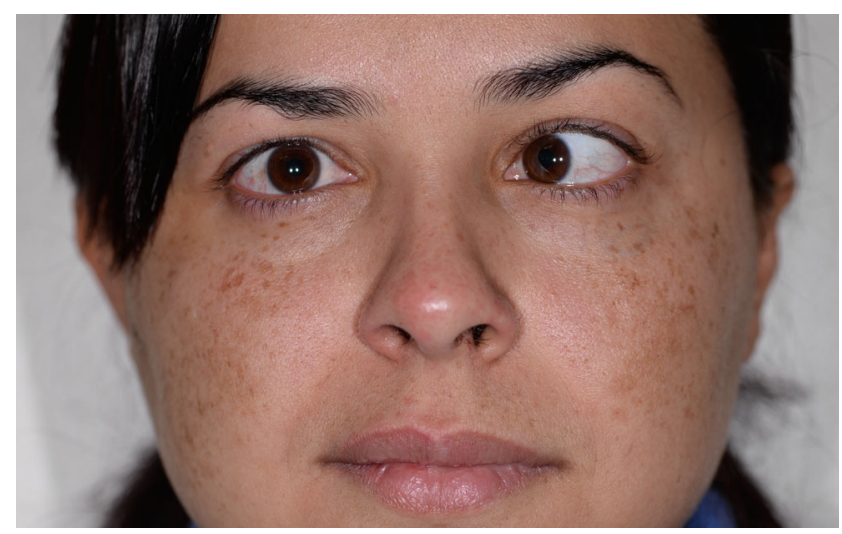

Fig. 2 Extraoral photo of the patient showing limitation of movement in dextroversion of the right eye

protocol was suggested to accelerate the healing of the tissue damaged, in association with vitamins B1 + B6 + B12 (Citoneurin ${ }^{\circledR}$, Merck SA, Rio de Janeiro, Brazil) for 14 days. Ophthalmology evaluation was requested on the same day after the surgical procedure; however, as we do not have this specialist in the hospital, evaluation was obtained after 4 days postoperatively. Ophthalmologist found maintenance of visual acuity (20/20) and suggested the continuous use of eye patch for 30 days and intermittent use only for solar exposure after this period.

The imaging of CTs showed that all the muscle, nerve, and bone structures were preserved without any abnormality. However, on the MRI, the presence of hyperintensity on T1weighted image was found running from the medial region of the TMJ to the region of the orbit, probably corresponding to the liquid leakage of the articular capsule, that may have compressed the nerve structures that resulted in paralysis of the abducens nerve of the right side (Figs. 3 and 4).

In the 30th day postoperatively, the patient reported improvement of diplopia, now only in levoversion. The clinical

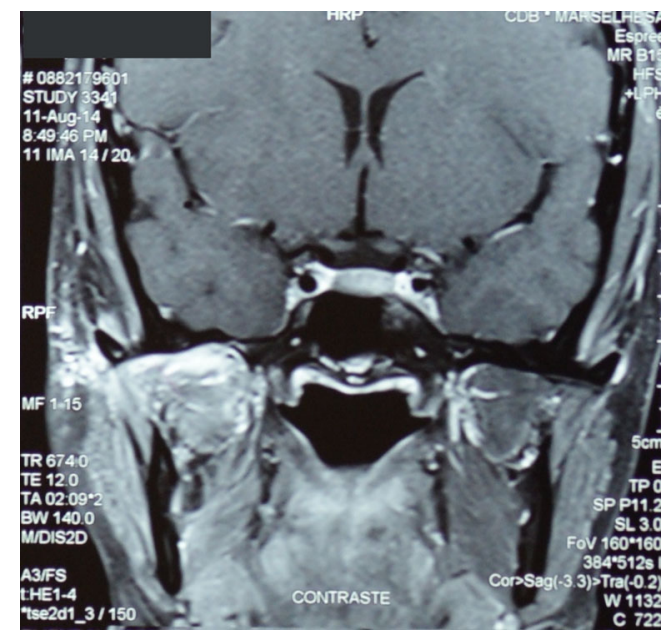

Fig. 3 Coronal view of the MRI showing hyperintensity from the medial wall of the right TMJ and extending medially 


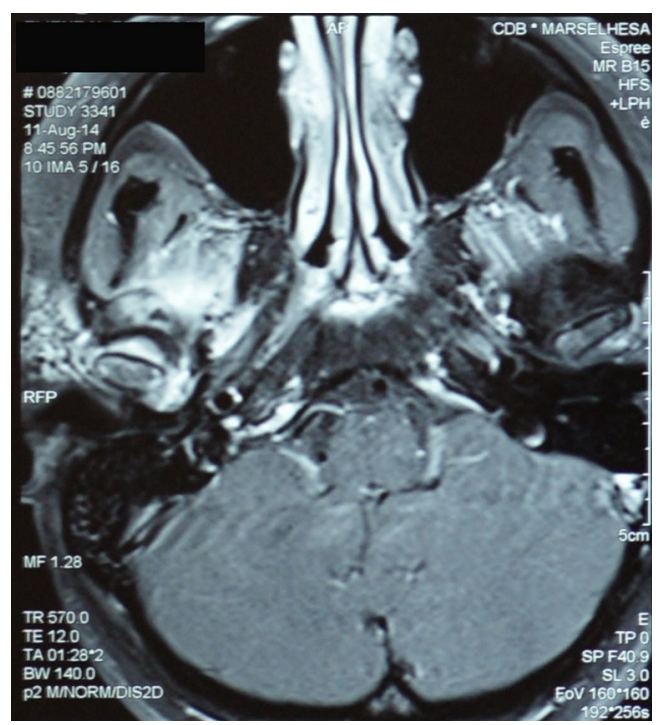

Fig. 4 Axial view of the MRI showing hyperintensity from the medial wall of the right TMJ and extending medially

examination of extrinsic ocular movement maintained the absence of abduction of the right eye, due to abducens nerve palsy. During this period, new exams were requested for the patient with an orbit CT showing preserved structures with exception of atrophy of the right lateral rectus muscle and mild of the abducens muscle resulted from their paralysis (Fig. 5).

After 75 postoperative days, patient reports important remission of the diplopia, maintaining mild medial displacement of the right eye without functional repercussions for the case (Figs. 6 and 7).

\section{Discussion}

Neurological lesions have a low incidence within the complications in arthroscopic procedures, ranging from 1.7 [8] to $1.5 \%$ [9]. Among these injuries to the nerves described in the literature, nerve injury to the fifth and seventh cranial nerves are the most common, with the fifth nerve injury

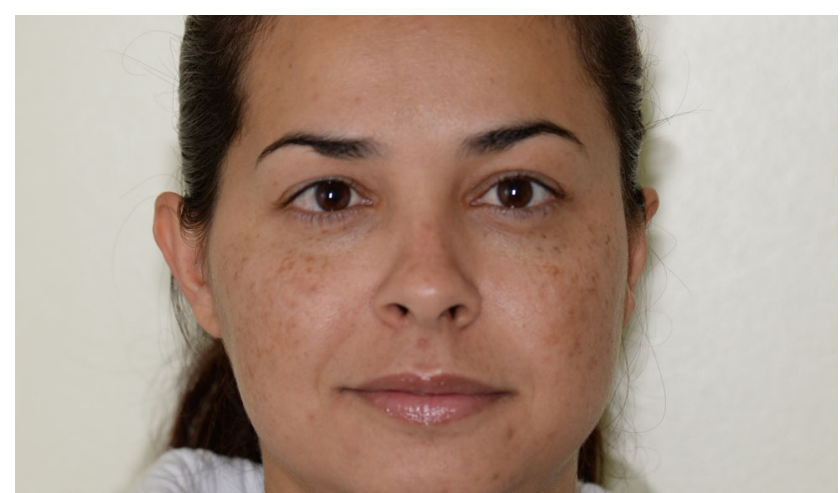

Fig. 6 Return of the right eye movement after 75 postoperative daysfront view

occurrence being the most common, mainly affecting the mandibular division of the trigeminal nerve, through the leakage of irrigation fluid by the medial capsule, and following the path along the mandibular branch with compression of their nerve endings in this region, as well as the inferior alveolar and lingual nerves. The injury to auriculotemporal nerve is also noteworthy, branch of the third division of the fifth cranial nerve, which innervates the TMJ region and can also be damaged directly by surgical manipulation. The seventh nerve injuries commonly occur by direct injury to the nerve during the trocar insertion for arthroscopic procedures.

Among the rare neurological complications after arthroscopic procedures, there is a description of a case of Horner's syndrome [10] and a case of paralysis of the oculomotor and trochlear nerves [2]. In the case of Horner's syndrome, the authors report that the patient developed ptosis and miosis in one eye, caused probably by leakage of fluid from the medial wall of the TMJ joint capsule, which migrated to the parapharyngeal space and led to carotid plexus compression. According to the case report that the patient presented paralysis of the oculomotor and trochlear nerves, the MRI showed a crack in the skull base, which led to an accumulation of fluid in the ipsilateral temporal lobe and cavernous sinus, with increased intracranial pressure, leading to paralysis of some cranial nerves on that side.
Fig. 5 CT showing atrophy of the right lateral rectus muscle

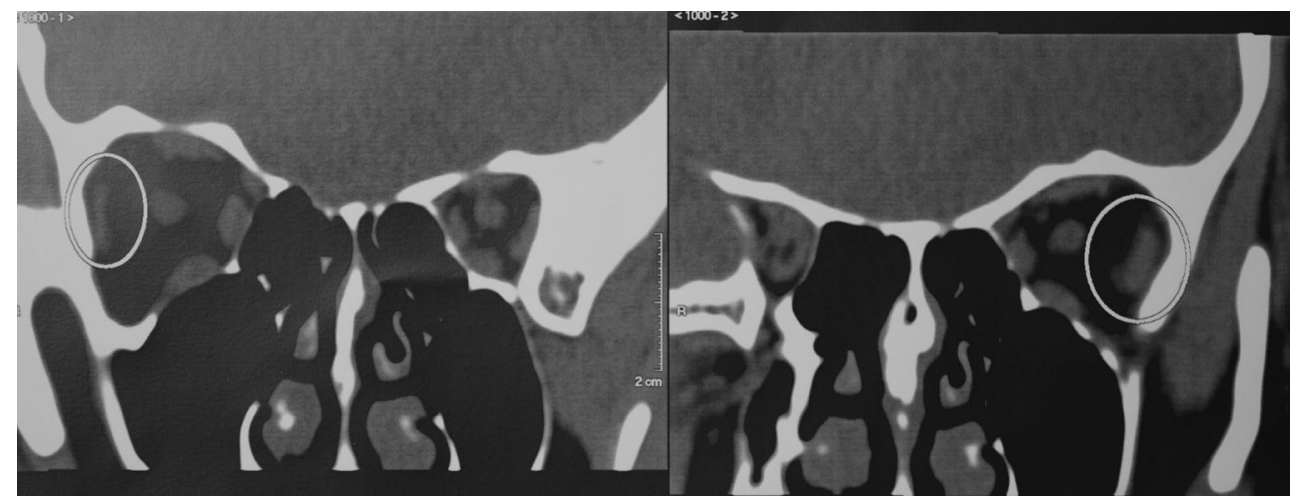




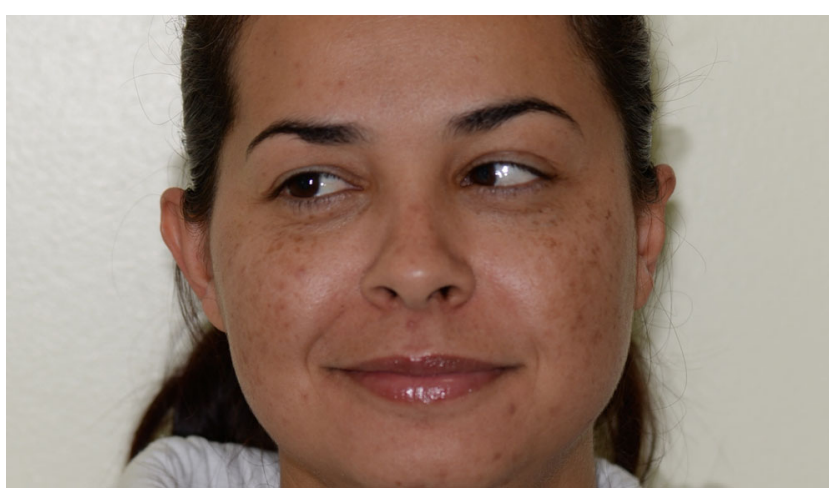

Fig. 7 Return of the right eye movement after 75 postoperative days in dextroversion

The abducens nerve arises from the adducens nucleus on the pons of the brain and exits the brainstem at the junction of the pons and the medulla. The nerve travels through the cavernous sinus at the tip of the petrous temporal bone and enters the orbit of the eye through the superior orbital fissure and terminates by innervating the lateral rectus muscle.

Through clinical and imaging data of our case, it is assumed that there probably must have been a leakage of fluid from the medial wall of the capsule, which affected the ipsilateral orbital fissure region, with compression and temporary injury to the abducens nerve at this level leading to a acute compartment syndrome, which after 2 months and a half regained its normal function. A hypothesis to this fluid leakage is that during the instrumentation, mechanical damage to the medial wall of the superior joint space may have happened with the use of the instruments, and due to the irrigation pressure, associated to the creation of a false path to the fluid outflow the TMJ capsule and goes to the lateral aspect of the orbit. No direct damage to the abducens nerve may have happened, as the instruments were not long enough to reach the nerve.

In summary, despite being a relatively safe procedure, care must be taken during the arthroscopic procedure, because as in this case, an accidental extravasation of irrigation fluid can result in a compartment syndrome framework, with injury to nerve structures that despite of being temporary, greatly affect the quality of life of patients in the immediately postoperative period.

Compliance with ethical standards Informed consent was obtained from the patient in this case report.

Conflict of interest The authors declare that they have no conflict of interest.

\section{References}

1. Zhang S, Yang C, Cai X, Liu X, Huang D, Xie Q, Haddad MS, Chen Z (2011) Prevention and treatment for the rare complications of arthroscopic surgery in the temporomandibular joint. J Oral Maxillofac Surg 69:e347-e353

2. Patel S, Jerjes W, Upile T, Hopper C (2010) TMJ arthroscopy: rare neurological complications associated with breach of the skull base. Br J Oral Maxillofac Surg 48:18-20

3. BR C, ALN C (2011) Optic, oculomotor, abducens, and facial nerve palsies after combined maxillary and mandibular osteotomy: case report. J Oral Maxillofac Surg 69:234-241

4. Kim JW, Chin BR, Park HS, Lee SH, Kwon TG (2011) Cranial nerve injury after Le Fort I osteotomy. Int J Oral Maxillofac Surg 40:327-329

5. Şirinoğlu H, Güvercin E, Çakmakoğlu Ç, Filinte GT, Yeșiloğlu N, Bozkurt M (2015) Idiopathic sixth nerve palsy after bimaxillary surgery: a complication or a sequential pathology independent from surgery? The Journal of Craniofacial Surgery 26:191-192

6. Pancko FX, Barrios TJ (2010) Post-traumatic bilateral abducens nerve palsy and unilateral facial nerve palsy: a case report. J Oral Maxillofac Surg 68:1694-1697

7. McCain JP, Podrasky AE, Zabiegalski NA (1992) Arthroscopic disc repositioning and suturing: a preliminary report. J Oral Maxillofac Surg 50:568-579

8. Tsuyama M, Kondoh T, Seto K, Fukuda J (2000) Complications of temporomandibular joint arthroscopy: a retrospective analysis of 301 lysis and lavage procedures performed using the triangulation technique. J Oral Maxillofac Surg 58:500-505

9. González-García R, Rodríguez-Campo FJ, Escorial-Hernández V, Muñoz-Guerra MF, Sastre-Pérez J, Naval-Gías L, Gil-Díez Usandizaga JL (2006) Complications of temporomandibular joint arthroscopy: a retrospective analytic study of 670 arthroscopic procedures. J Oral Maxillofac Surg 64:1587-1591

10. Martín-Moro JG, Sastre-Pérez J, Pena Fernández I (2009) Horner syndrome after temporomandibular joint arthroscopy: a new complication. J Oral Maxillofac Surg 67:1320-1322 